\title{
Rapidly progressive sensory peripheral neuropathy secondary to Coxiella burnetii infection
}

\author{
Adriana Octaviana Dulamea ${ }^{1,2}$, Ioan-Cristian Lupescu' ${ }^{1}$ \\ ${ }^{1}$ Neurology Department, Fundeni Clinical Institute, Bucharest, Romania \\ 2"Carol Davila" University of Medicine and Pharmacy, Bucharest, Romania
}

\begin{abstract}
$Q$ fever is a zoonosis produced by Coxiella burnetii, with potential neurological complications such as aseptic meningitis, myelitis, encephalitis and peripheral neuropathy. We report the case of a 68-year-old male with recent history of acute $Q$ fever, who developed progressive numbness of the hands, lower limbs and abdomen. Clinical exam revealed bilateral crural loss of proprioception and vibration senses, positive Romberg, bilateral Babinski and intact DTRs. Initial electroneurography was normal, however, when repeated, revealed axonal sensory polyneuropathy. Brain and cord MRI, including assessment of lumbo-sacral plexus, was also normal. All laboratory findings were within normal range, except for slightly elevated glycemia. Plasma exchange was initially tried producing limited improvement. Repeated C.burnetii serology and CSF analysis revealed elevated levels of IgM and IgG, still suggestive of acute infection. For this reason, the patient continued treatment with Doxycycline and upon discharge from hospital, finally reported improvement of symptoms, proving retrospectively that the neuropathy was secondary to C.burnetii infection.
\end{abstract}

Keywords: Coxiella burnetii, sensory neuropathy, plasma exchange

Abbreviations (in alphabetical order):

CSF - Cerebrospinal fluid

DTR - Deep tendon reflexes

IgM - Immunoglobulin M
IgG - Immunoglobulin G

MRI - Magnetic Resonance Imaging

\section{INTRODUCTION}

Q fever is a zoonosis caused by Coxiella burnet$i$, that has a wide variety of clinical presentations (1). It can range from asymptomatic seroconversion, to acute illness (usually in the form of a flulike syndrome, pneumonia and/or hepatitis) or chronic illness (mainly endocarditis) (2).

Neurological complications have also been described and include aseptic meningitis, encephalitis, myelitis and peripheral neuropathy (3).

\section{CASE REPORT}

We present the case of a 68-year-old male, non-smoker, non-alcohol consumer, who was ad- mitted for numbness and pain with electric shock sensations of lower limbs, upper limbs, abdomen and in the spine upon flexion of the head (Lhermitte sign). Onset was 3 weeks prior, initially affecting only the feet, but with ascendant progression. The patient also accused paresthesias of both hands.

Medical history revealed type II diabetes mellitus controlled with diet and a diagnosis of acute $\mathrm{Q}$ Fever 3 months prior (positive serology for Coxiella burnetii IgM antibodies).

Neurological exam highlighted loss of vibration and position senses in lower limbs, positive Romberg, intact DTRs, bilateral Babinski and orthostatic hypotension. 
Initial electroneurography was normal. Brain MRI showed brain microangiopathy and spinal cord MRI was normal ruling out cervical myelopathy or demyelinating disease.

Laboratory findings revealed elevated glycaemia and glycated hemoglobin. Auto-immune and tumor markers, vitamin B12, infectious markers (hepatitis $\mathrm{B}$ and $\mathrm{C}$ viruses, human immunodeficiency virus, syphilis, Borrelia), serum free light chains and urine porphyrins were all within normal range.

A second electroneurography indicated axonal sensory polyneuropathy (normal sensory conduction velocities and diminished sensory nerve action potentials on the right superficial peroneal nerve and bilateral median and ulnar nerves).

CSF analysis showed a slight increase in proteins $(0.4 \mathrm{~g} / \mathrm{L})$.

Given the rapid progression of symptoms, the polyneuropathy was considered secondary to C.burnetii infection and the patient received Doxycycline $100 \mathrm{mg}$ b.i.d. for 14 days (4-5).

However, symptoms persisted. We repeated C.burnetii serology and CSF analysis, which showed IgM and IgG elevated titers, still suggestive for acute infection.

Coxiella burnetii serology

\begin{tabular}{|l|c|c|}
\hline & Result & Normal range \\
\hline IgG phase I & $1 / 512$ & $<1 / 16$ \\
\hline IgM phase I & $1 / 1024$ & $<1 / 16$ \\
\hline IgG phase II & $1 / 16000$ & $<1 / 16$ \\
\hline IgM phase II & $1 / 32$ & $<1 / 16$ \\
\hline
\end{tabular}

The most probable mechanism was an immunologic reaction triggered by bacterial antigens through molecular mimicry.

An infectious disease specialist was contacted and recommended Doxycycline $100 \mathrm{mg}$ b.i.d. for 30 days and Ciprofloxacin $500 \mathrm{mg}$ b.i.d. for 14 days, for preventing chronicity of infection during immunosuppressive treatment.

Knowing that endocarditis can complicate Q fever (6-7), we performed an echocardiography, which was within normal range.

Pulse therapy with Methylprednisolone 1,000 $\mathrm{mg}$ iv daily for 3 days was initially tried, but didn't improve symptoms.

Plasma exchange was then performed. Electroneurography done after plasmapheresis revealed normal sensory and motor conduction velocities. However, there was no neurological improvement of sensory deficit at lower limbs.

We repeated MRI of thoraco-lumbar cord with analysis of lumbo-sacral plexus and Gadolinium injection, but no lesions were detected.

In this setting, the only option was to continue treatment with Doxycycline in the hope of clearing the infection. Mirtazapine $15 \mathrm{mg}$ q.d. was added for the patient's anxiety and Gabapentine $300 \mathrm{mg}$ q.d was administered for neuropathic pain management..

Upon finishing antibiotic treatment, the patient finally reported improvement of symptoms, thus proving retrospectively that the neuropathy was most likely secondary to C.burnetii infection.

\section{DISCUSSION AND CONCLUSIONS}

The bilateral distal-to-proximal progression of numbness suggested a length-dependent polyneuropathy with rapid progression. Loss of vibration and position sense pointed to involvement of large myelinated fibers. However, the electric shock like sensations appearing several times a day in the limbs and the normal sensory and motor velocities obtained at the first electromyography may suggest a small fiber neuropathy that could not be proved for certain because patient refused skin biopsy.

The clinical picture was complicated by intact DTRs and bilateral pyramidal syndrome.

Brain and cord MRI showed only brain microangiopathy and excluded cervical myelopathy and demyelinating disease, while electromyogram, CSF analysis and MRI of lumbo-sacral plexus didn't confirm the presence of polyradiculoneuropathy.

Although the patient presented type II diabetes mellitus, the clinical picture was not characteristic for diabetic neuropathy.

Electroneurographic improvement of conduction velocities and clinical neurological improvement after plasma exchange and specific antibiotic therapy confirmed the infectious nature of the neuropathy. 


\section{REFERENCES}

1. Raoult D., Marrie T., Mege J. Natural history and pathophysiology of Q fever. Lancet Infect Dis. 2005; 5(4):219-226.

2. Hartzell J.D., Wood-Morris R.N., Martinez L.J. et al. Q fever: epidemiology, diagnosis, and treatment. Mayo Clin Proc. 2008; 83(5):574-579.

3. Bernit E., Pouget J., Janbon F. et al. Neurological involvement in acute $Q$ fever: a report of 29 cases and review of the literature. Arch Intern Med. 2002; 162(6):693-700.

4. Anderson A., Bijlmer H., Fournier P.E. et al. Diagnosis and management of $Q$ fever - United States, 2013: recommendations from CDC and the Q Fever Working Group. MMWR Recomm Rep. 2013; 62(RR03):1-23

5. Brouillard J.E., Terriff C.M., Tofan A. et al. Antibiotic selection and resistance issues with fluoroquinolones and doxycycline against bioterrorism agents. Pharmacotherapy. 2006; 26(1):3-14.

6. Hartzell J.D., Wood-Morris R.N., Martinez L.J. et al. Q fever: epidemiology, diagnosis, and treatment. Mayo Clin Proc. 2008; 83(5):574-579.

7. Karakousis P.C., Trucksis M., Dumler J.S. Chronic Q Fever in the United States. J Clin Microbiol. 2006; 44(6):2283-2287. 